Journal of

Applied

Crystallography

ISSN 0021-8898

Received 12 November 2001

Accepted 8 May 2002

\section{Three-dimensional Pt-nanoparticle networks studied by anomalous small-angle $X$-ray scattering and $X$-ray absorption spectroscopy}

\author{
T. Vad, ${ }^{a *}$ H.-G. Haubold, ${ }^{a}$ N. Waldöfner ${ }^{b}$ and H. Bönnemann ${ }^{b}$ \\ a Institut für Festkörperforschung, Forschungszentrum Jülich GmbH, D-52425 Jülich, Germany, and \\ ${ }^{\text {b} A b t e i l u n g ~ H e t e r o g e n e ~ K a t a l y s e, ~ M a x-P l a n c k-I n s t i t u t ~ f u ̈ r ~ K o h l e n f o r s c h u n g, ~ D-45466 ~ M u ̈ l h e i m ~ a n ~}$ \\ der Ruhr, Germany. Correspondence e-mail: th.vad@fz-juelich.de
}

\begin{abstract}
Anomalous small-angle X-ray scattering (ASAXS) experiments with synchrotron radiation were performed to study the three-dimensional nanostructures of metal/organic hybrids formed by crosslinking aluminium-organic-stabilized platinum nanoparticles with various bifunctional organic spacer molecules. The advantage of ASAXS is the possibility of separating the particle scattering from that of the organic components, thus providing unbiased information about particle size distributions and interparticle correlation. In order to obtain the structural information from the scattering data, a model function based on Vrij's analytical solution for a multicomponent system of hard spheres is proposed. The model is applied to three different samples and the results are compared with those obtained from the application of Fourier methods (characteristic function) and X-ray absorption measurements.
\end{abstract}

(C) 2002 International Union of Crystallography Printed in Great Britain - all rights reserved low-molecular matrix, the particle scattering intensity can be written as

$$
\frac{\mathrm{d} \Sigma}{\mathrm{d} \Omega}(Q)=c_{0} n^{2} f_{0}^{2} S^{2}(Q) V^{2},
$$

where $c_{0}=N_{\text {particle }} / V_{\text {sample }}$ is the number density of the particles, $n=N_{\text {Atoms }} / V$ ( $V=$ particle volume $)$ is the number density of the metal atoms within a particle, $f_{0}$ is the scattering amplitude, and $S^{2}(Q)$ is the intraparticle interference factor, i.e. the form factor of a particle.

For a spherical particle of radius $R_{0}$, the particle form factor is defined by

$$
S(Q)=3 \frac{\sin \left(Q R_{0}\right)-Q R_{0} \cos \left(Q R_{0}\right)}{\left(Q R_{0}\right)^{3}},
$$

with the magnitude of the scattering vector $Q=4 \pi \sin (\Theta) / \lambda$ ( $\lambda$ is the $\mathrm{X}$-ray wavelength, $2 \Theta$ is the scattering angle).

1.1.2. Anomalous small-angle $X$-ray scattering. In order to obtain more information on the (pure) particle scattering, it is convenient to perform anomalous small-angle X-ray scattering (ASAXS) experiments (for a detailed description of the ASAXS theory see, e.g. Haubold et al., 1994; Haubold \& Wang, 1995; Materlik et al., 1994). For the two-phase model, the total differential cross section is given by

$$
\frac{\mathrm{d} \Sigma}{\mathrm{d} \Omega}(Q)=c_{0}\left(n_{p} f_{p}-n_{m} f_{m}\right)^{2} S^{2}(Q) V^{2}+\frac{\mathrm{d} \Sigma}{\mathrm{d} \Omega_{\mathrm{bg}}}(Q),
$$

where $n_{p}, f_{p}, n_{m}, f_{m}$ are the number densities and atomic form factors, respectively, of the particles $(p)$ and the surrounding matrix $(m)$. Besides the particle scattering, an additional background scattering term $\mathrm{d} \Sigma / \mathrm{d} \Omega_{\mathrm{bg}}(Q)\left(\propto Q^{-4}\right.$ in most 
cases) contributes to the total differential cross section, which, for the most part, originates from inhomogeneities in the matrix (Porod, 1951). Therefore, the SAXS intensity contains almost always a (in many cases non-negligible) contribution from the matrix if a SAXS experiment is performed at only one energy. Fortunately, the particle scattering can quite easily be separated from the matrix background if, e.g. for platinum particles, contrast-variation experiments at (at least) two different energies near the $\mathrm{Pt} L_{3}$-absorption edge $\left[E\left(\mathrm{Pt} L_{3}\right)=\right.$ $11.564 \mathrm{keV}$ ] are carried out (see example with simulated data, Fig. 1).

Since, for our case, the atomic form factors

$$
f(E)=f_{0}+f^{\prime}(E)+i f^{\prime \prime}(E)
$$

of the mainly organic matrix components $(\mathrm{C}, \mathrm{H}, \mathrm{O})$ remain almost constant in this energy regime (see Fig. 2) the simple subtraction of two SAXS curves measured at two different energies $E_{1}$ and $E_{2}$ yields the (almost) pure (Pt) metal-particle scattering

$$
\begin{aligned}
\frac{\mathrm{d} \Sigma}{\mathrm{d} \Omega_{p}}(Q)= & \frac{\mathrm{d} \Sigma}{\mathrm{d} \Omega}\left(Q, E_{1}\right)-\frac{\mathrm{d} \Sigma}{\mathrm{d} \Omega}\left(Q, E_{2}\right) \\
= & c_{0} n^{2}\left[f_{\mathrm{Pt}}^{2}\left(E_{1}\right)-f_{\mathrm{Pt}}^{2}\left(E_{2}\right)\right] S^{2}(Q) V^{2} \\
& \times\left\{1-\frac{2 n_{m} f_{m}}{n\left[f_{\mathrm{Pt}}\left(E_{1}\right)+f_{\mathrm{Pt}}\left(E_{2}\right)\right]}\right\}
\end{aligned}
$$

(in the case of negligible $f^{\prime \prime}$ correction terms), which reduces to

$$
\begin{aligned}
\frac{\mathrm{d} \Sigma}{\mathrm{d} \Omega_{p}}(Q) & =c_{0} n^{2}\left[f_{\mathrm{Pt}}^{2}\left(E_{1}\right)-f_{\mathrm{Pt}}^{2}\left(E_{2}\right)\right] S^{2}(Q) V^{2} \\
& =c_{0} \Delta(n f)^{2} S^{2}(Q) V^{2}
\end{aligned}
$$

if $2 n_{m} f_{m} /\left\{n\left[f_{\mathrm{Pt}}\left(E_{1}\right)+f_{\mathrm{Pt}}\left(E_{2}\right)\right]\right\} \ll 1$.

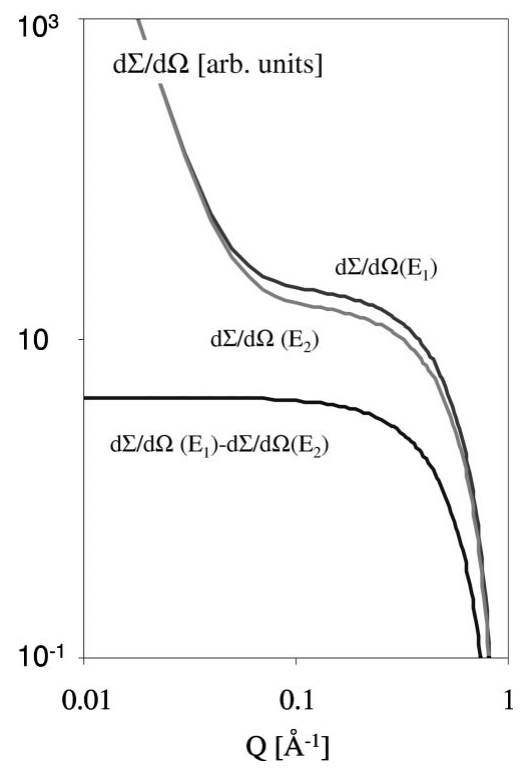

Figure 1

Simulated SAXS intensities $\mathrm{d} \Sigma / \mathrm{d} \Omega$ for a sphere of radius $R=5 \AA$ with energy-dependent scattering density and additional energy-independent $Q^{-4}$ background at energies $E_{1}, E_{2}$, and separated particle scattering $\mathrm{d} \Sigma / \mathrm{d} \Omega\left(E_{1}\right)-\mathrm{d} \Sigma / \mathrm{d} \Omega\left(E_{2}\right)$.
1.1.3. Interparticle interference. At higher particle concentrations, the effect of interparticle interference has also to be taken into account, leading to

$$
\frac{\mathrm{d} \Sigma}{\mathrm{d} \Omega_{p}}(Q)=c_{0} \Delta(n f)^{2} S^{2}(Q) V^{2}[1+H(Q)],
$$

with

$$
H(Q)=c_{0} \int_{0}^{\infty} 4 \pi r^{2} h(r) \frac{\sin (Q r)}{Q r} \mathrm{~d} r, \quad h(r)=g(r)-1,
$$

where $h(r)$ is the total correlation function and $g(r)$ the radial distribution function as a function of $r$, the distance between the force centres of two particles.

A well known case is the hard-spheres repulsion

$$
U(r)= \begin{cases}\infty & r<2 R_{0} \\ 0 & r \geq 2 R_{0}\end{cases}
$$

which is a good approximation for a fluid consisting of particles with a steep repulsive core, such as metal particles.

1.1.4. Mixtures of hard spheres - Vrij's equations. The case becomes more complicated as the particle sizes are not uniform. For a mixture of hard spheres with $p$ different particle radii, the scattering is given by Fournet's expression (Fournet, 1955)

$$
\begin{aligned}
\frac{\mathrm{d} \Sigma}{\mathrm{d} \Omega_{p}}(Q)= & \sum_{i=1}^{p} \sum_{j=1}^{p}\left(c_{i} c_{j}\right)^{1 / 2} n_{i} f_{i} n_{j} f_{j} S_{i}(Q) S_{j}(Q) V_{i} V_{j} \\
& \times\left[\delta_{i j}+H_{i j}(Q)\right],
\end{aligned}
$$

where $c_{i}$ is the number of particles of radius $R_{i}$ per unit volume, and $\delta_{i j}$ is the Kronecker delta.

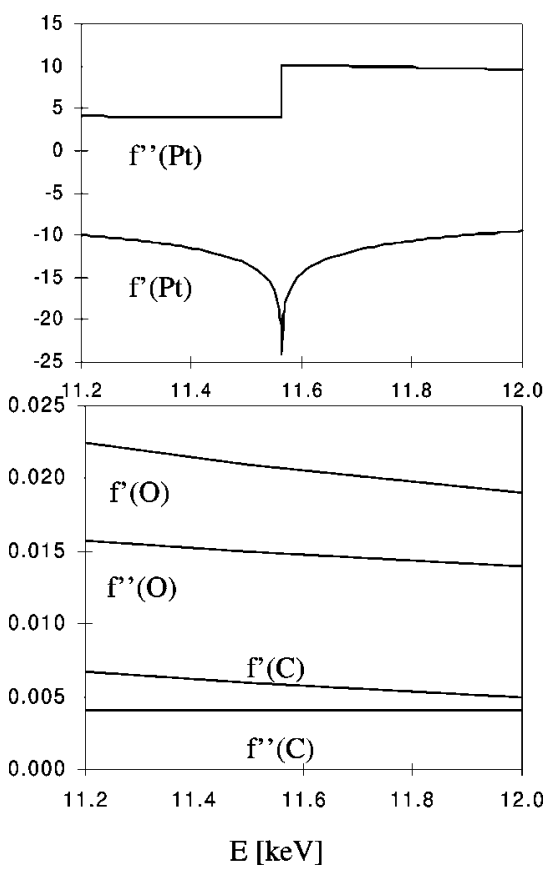

Figure 2

Anomalous dispersion corrections $f^{\prime}, f^{\prime \prime}$ for $\mathrm{Pt}, \mathrm{C}$ and $\mathrm{O}$ in the vicinity of the Pt $L_{3}$-absorption edge (after Cromer \& Liberman, 1981). 
For particles of constant density, equation (10) reduces to

$$
\begin{aligned}
\frac{\mathrm{d} \Sigma}{\mathrm{d} \Omega_{p}}(Q)= & \left(n f_{0}\right)^{2} \sum_{i=1}^{p} \sum_{j=1}^{p}\left(c_{i} c_{j}\right)^{1 / 2} S_{i}(Q) S_{j}(Q) V_{i} V_{j} \\
& \times\left[\delta_{i j}+H_{i j}(Q)\right]
\end{aligned}
$$

and $\left(n f_{0}\right)^{2} \rightarrow \Delta(n f)^{2}$ in the case of an ASAXS experiment. The $H_{i j}(Q)$ are defined by

$$
\begin{aligned}
H_{i j}(Q) & =\left(c_{i} c_{j}\right)^{1 / 2} \int_{0}^{\infty} 4 \pi r^{2} h_{i j}(r) \mathrm{d} r \\
& =\left(c_{i} c_{j}\right)^{1 / 2} \int_{0}^{\infty} 4 \pi r^{2}\left[g_{i j}(r)-1\right] \mathrm{d} r .
\end{aligned}
$$

Another description of the scattering from a mixture of hard spheres is possible by employing the Fourier transforms $C_{i j}(Q)$ of the direct correlation functions $\zeta_{i j}(r)$. The $C_{i j}(Q)$ are defined by the multicomponent Ornstein-Zernike (OZ) relation (Baxter, 1970)

$$
h_{i j}(r)=\zeta_{i j}(r)+\sum_{k=1}^{p} c_{k} \int \zeta_{i k} h_{k j}\left(\left|\mathbf{r}-\mathbf{r}^{\prime}\right|\right) \mathrm{d} \mathbf{r}^{\prime} .
$$

Multiplying both sides of the $\mathrm{OZ}$ relation by $\left(c_{i} c_{j}\right)^{1 / 2} \exp (i \mathbf{Q} \cdot \mathbf{r})$ and integrating with respect to $\mathbf{r}$ over all three-dimensional space, one obtains the matrix equation

$$
(\mathbf{I}+\mathbf{H})(\mathbf{I}-\mathbf{C})=\mathbf{I},
$$

where $\mathbf{I}=\left[\delta_{i j}\right]$ is the identity matrix. Since

$$
(\mathbf{I}-\mathbf{C})^{-1}=\frac{1}{\operatorname{det}(\mathbf{I}-\mathbf{C})}(\mathbf{I}-\mathbf{C})^{T}
$$

one obtains

$$
\delta_{i j}+H_{i j}(Q)=\frac{1}{[\Delta(Q)]}|\mathbf{I}-\mathbf{C}(Q)|^{j i},
$$

where $[\Delta(Q)]=\operatorname{det}[\mathbf{I}-\mathbf{C}(Q)]$ and $|\mathbf{I}-\mathbf{C}(Q)|^{j i}$ is the cofactor of the matrix element $\delta_{i j}-C_{i j}(Q)$. Hence, the (A)SAXS intensity can be written as

$$
\frac{\mathrm{d} \Sigma}{\mathrm{d} \Omega_{p}}(Q)=-\left[D_{f}(Q)\right][\Delta(Q)]^{-1},
$$

where the $(p+1) \times(p+1)$ determinant $\left[D_{f}(Q)\right]$ is given by

$$
\left[D_{f}(Q)\right]=\Delta(n f)^{2}\left|\begin{array}{cc}
0 & c_{j}^{1 / 2} S_{j}(Q) V_{j} \\
c_{i}^{1 / 2} S_{i}(Q) V_{i} & \delta_{i j}-C_{i j}(Q)
\end{array}\right| .
$$

Among others (Blum \& Stell, 1979; Salacuse \& Stell, 1982), it was Vrij (1978, 1979) who showed that the scattering equation for a mixture of hard spheres can be represented by a closed expression for any number of hard spheres. For the determinant $[\Delta(Q)]$, Vrij obtained

$$
[\Delta(Q)]=\left(F_{11} F_{22}-F_{12} F_{21}\right)\left(F_{11}^{*} F_{22}^{*}-F_{12}^{*} F_{21}^{*}\right)\left(1-\xi_{3}\right)^{-4},
$$

with

$$
\begin{aligned}
& F_{11}(Q)=1-\xi_{3}+\left\langle d^{3} \exp (i X) \Phi\right\rangle \\
& F_{12}(Q)=\left\langle d^{4} \exp (i X) \Phi\right\rangle \\
& F_{22}(Q)=1-\xi_{3}+3\left\langle d^{3} \exp (i X) \Psi\right\rangle \\
& F_{21}(Q)=\frac{1}{2}\left(1-\xi_{3}\right) i Q-3 \xi_{2}+3\left\langle d^{2} \exp (i X) \Psi\right\rangle
\end{aligned}
$$

and $F_{i j}(Q)=F_{i j}(-Q)$. The quantities $\xi_{v}, X, \Phi, \Psi$, $\left\langle d^{v} \exp (i X) \Phi\right\rangle$ and $\left\langle d^{v} \exp (i X) \Psi\right\rangle$ are defined by

$$
\xi_{v}=\frac{\pi}{6} \sum_{j=1}^{p} c_{j} d_{j j}^{v}
$$

where $d_{j j}$ is the distance between two particles of the same radius $R_{j}$,

$$
\begin{gathered}
X_{j}=\frac{1}{2} Q d_{j j}, \\
\Phi\left(X_{j}\right)=3 \frac{\sin \left(X_{j}\right)-X_{j} \cos \left(X_{j}\right)}{X_{j}^{3}}, \\
\Psi\left(X_{j}\right)=\frac{\sin \left(X_{j}\right)}{X_{j}}, \\
\left\langle d^{v} \exp (i X) \Phi\right\rangle=\frac{\pi}{6} \sum_{j=1}^{p} c_{j} d_{j j}^{v} \exp \left(i X_{j}\right) \Phi\left(X_{j}\right),
\end{gathered}
$$

and

$$
\left\langle d^{v} \exp (i X) \Psi\right\rangle=\frac{\pi}{6} \sum_{j=1}^{p} c_{j} d_{j j}^{v} \exp \left(i X_{j}\right) \Psi\left(X_{j}\right) .
$$

The determinant $-\left[D_{f}(Q)\right]$ is given by

$$
\begin{aligned}
-\left[D_{f}(Q)\right]= & {\left[\left\langle\Delta(n f)^{2} S^{2}(Q) V^{2}(R)\right\rangle T_{1} T_{1}^{*}\right.} \\
& +\left\langle d^{6} \Phi^{2}\right\rangle T_{2} T_{2}^{*}+9\left\langle d^{4} \Psi^{2}\right\rangle T_{3} T_{3}^{*} \\
& +\left\langle\Delta(n f) S(Q) V(R) d^{3} \Phi\right\rangle \\
& \times\left(T_{1} T_{2}^{*}+T_{1}^{*} T_{2}\right) \\
& +3\left\langle\Delta(n f) S(Q) V(R) d^{2} \Psi\right\rangle \\
& \times\left(T_{1} T_{3}^{*}+T_{1}^{*} T_{3}\right) \\
& \left.+3\left\langle d^{5} \Phi \Psi\right\rangle\left(T_{2} T_{3}^{*}+T_{2}^{*} T_{3}\right)\right] \\
& \times\left[(6 / \pi)\left(1-\xi_{3}\right)^{-4}\right]
\end{aligned}
$$

with

$$
\begin{aligned}
T_{1}= & F_{11} F_{22}-F_{12} F_{21}, \\
T_{2}= & F_{21}\langle d \Delta(n f) S(Q) V(R) \exp (i X)\rangle \\
& -F_{22}\langle\Delta(n f) S(Q) V(R) \exp (i X)\rangle, \\
T_{3}= & F_{12}\langle\Delta(n f) S(Q) V(R) \exp (i X)\rangle \\
& -F_{11}\langle d \Delta(n f) S(Q) V(R) \exp (i X)\rangle .
\end{aligned}
$$

1.1.5. Pt-metal-particle networks. In our special case of a three-dimensional Pt-metal-particle network, the particles are assumed to be interconnected by organic spacer molecules of uniform length $l_{\mathrm{sp}}$. In addition, the particles are surrounded by an organic protecting shell. Since the molecules forming the shell do not exhibit a size distribution, the thickness $t_{\mathrm{ps}}$ is also 
assumed to be a constant (see Fig. 3). The distance $d_{j j}$ between two particles of radius $R_{j}$ is then defined by

$$
d_{j j}=2 R_{j}+2 t_{\mathrm{ps}}+l_{\mathrm{sp}} .
$$

In a least-squares refinement procedure, the quantities $t_{\mathrm{ps}}$ and $l_{\text {sp }}$ cannot be simultaneously refined, since the correlation between these two parameters would be too large. In order to circumvent the effect of parameter correlation, we therefore introduce the sum $D_{0}=2 t_{\mathrm{ps}}+l_{\mathrm{sp}}$, i.e. the distance between the surfaces of two metal particles, so that the total interparticle distance $d_{j j}$ is given by

$$
d_{j j}=2 R_{j}+D_{0}
$$

This approach is the same as the one used by Pedersen (1994) for the analysis of silica particles coated with octadecyl chains (de Kruif et al., 1988): the 'hard-sphere' radius $R_{\mathrm{HS}}$, i.e. the half-distance between two particles of radius $R$, is given by the sum of the metal-particle radius $R$ and an additional constant $\Delta R$, which is independent of the particle size distribution $\left(R_{\mathrm{HS}}=d / 2=R+\Delta R\right)$.

Another approach, which might have been used, is to describe the hard-sphere radius as the product $R_{\mathrm{HS}}=C R$, where $C$ is a constant. Here, it is assumed that the size of the surrounding shell is proportional to the particle size. This is the case, e.g. for spherical precipitates in alloys, where the size of the depleted zone is proportional to the size of the precipitate (Pedersen, 1993; Sequeira et al., 1995; Pedersen et al., 1996). In the present study, however, this approach is unfavourable, since there is no physical reason why the thickness of the protecting shell (and/or the length of the spacer molecules) should depend on the particle size.
Transmission electron microscopy (TEM) investigations of pure Pt colloids have shown that the particle size distributions of the metal particles are well described by a (continuous) lognormal distribution (Fig. 3), i.e.

$$
N(R) \propto P(R)=\frac{1}{(2 \pi)^{1 / 2} \sigma_{0} R} \exp \left[-\frac{\ln ^{2}\left(R / R_{0}\right)}{2 \sigma_{0}^{2}}\right],
$$

where $\sigma_{0}$ is a parameter defining the full width at halfmaximum of the distribution, and $R_{0}$ is the most frequently occurring radius. Therefore,

$$
c_{i} \rightarrow d c\left(R_{i}\right)=c_{0} P\left(R_{i}\right) \mathrm{d} R
$$

where $c_{0}=N_{0} / V_{\text {sample }}$ is the number density of all particles in the sample,

$$
\begin{gathered}
\xi_{v}=c_{0} \frac{\pi}{6} \int_{0}^{\infty} P(R) d^{v} \mathrm{~d} R, \\
\left\langle d^{v} \exp (i X) \Phi\right\rangle=c_{0} \frac{\pi}{6} \int_{0}^{\infty} P(R) d^{v} \exp (i X) \Phi(X) \mathrm{d} R, \\
\left\langle d^{v} \exp (i X) \Psi\right\rangle=c_{0} \frac{\pi}{6} \int_{0}^{\infty} P(R) d^{v} \exp (i X) \Psi(X) \mathrm{d} R,
\end{gathered}
$$

and $X=X(R)=Q\left(2 R+D_{0}\right) / 2, d^{v}=d^{\nu}(R)=\left(2 R+D_{0}\right)^{v}$. Thus, the particle scattering is completely described by a fiveparameter model function
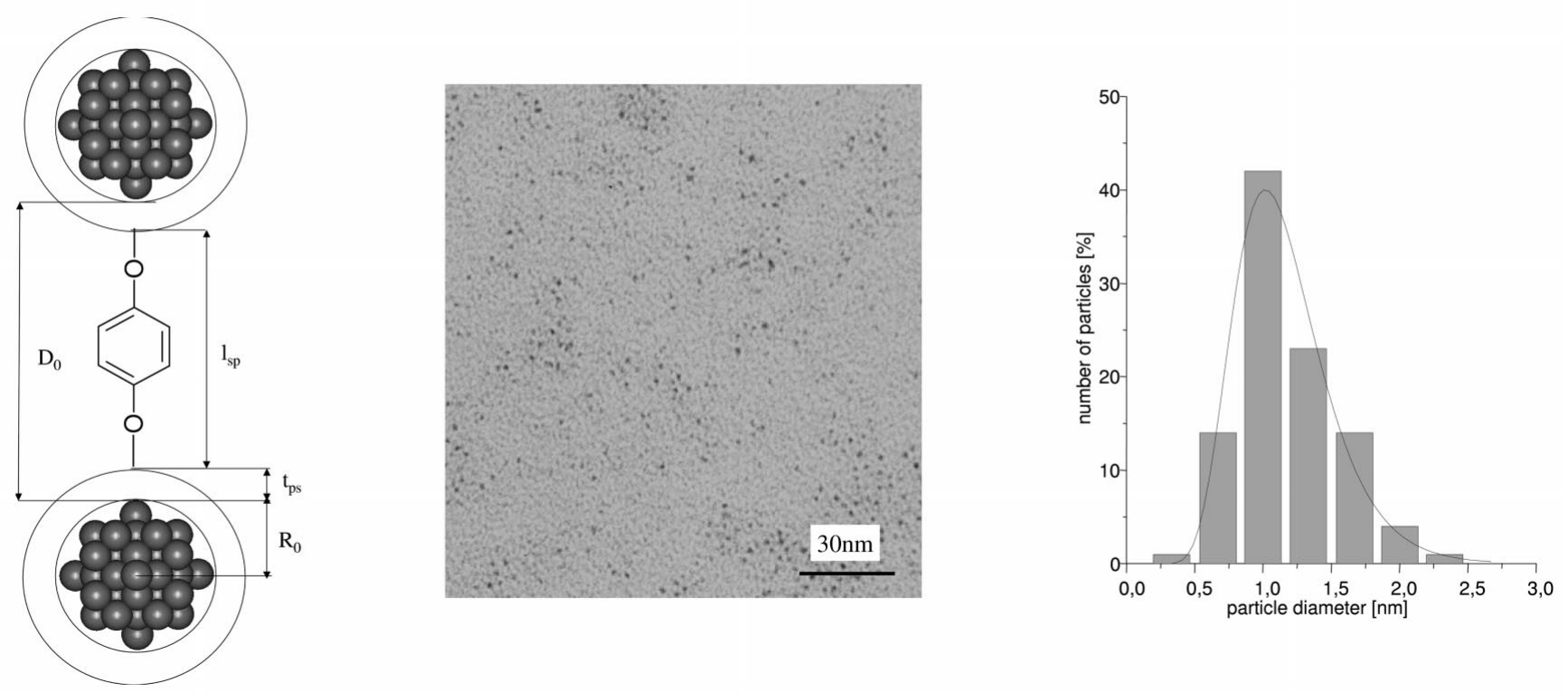

Figure 3

Definition of the parameters $D_{0}, l_{\mathrm{sp}}$ and $t_{\mathrm{ps}}$ (left), TEM image of a dilute dispersion of a Pt colloid powder in tetrahydrofuran (middle), and corresponding particle size distribution (right): the bar-chart is the result from the TEM analysis; the solid line is a log-normal size distribution for the particle diameters $D_{p}\left(=2 R_{p}\right)$ showing a maximum at $R_{0}=5.3 \AA$. 


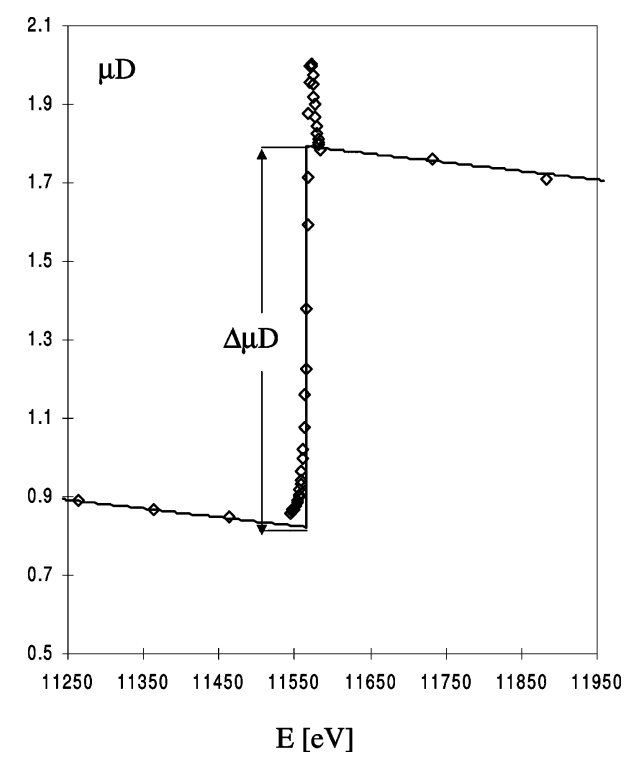

Figure 4

Edge jump of a Pt colloid at the Pt $L_{3}$-absorption edge. The solid line is the result from a fit of pre-edge and post-edge data of the absorption spectrum.

$$
\frac{\mathrm{d} \Sigma}{\mathrm{d} \Omega_{p}}(Q)=F\left[Q, \Delta(n f), c_{0}, \sigma_{0}, R_{0}, D_{0}\right]
$$

where the scattering contrast $\Delta(n f)$ is an additional parameter if the densities of the particles and/or the matrix are unknown.

1.1.6. X-ray absorption spectroscopy (XAS). In the study presented here, the main reason for recording X-ray absorp- tion spectra for each sample is to locate each individual absorption edge (which may vary considerably with chemical bonding) most exactly, since this knowledge is essential for the successful performance of a contrast-variation experiment (see previous section). Moreover, one obtains additional information on the Pt-metal content in the sample, and the valence state of the Pt atoms, by application of rather simple methods.

1.1.7. Determination of the Pt loading. From the measured edge jump,

$$
\Delta \mu D=\mu D\left(E_{+}\right)-\mu D\left(E_{-}\right)
$$

in the absorption spectrum (see Fig. 4) and the theoretical edge jump for free $\mathrm{Pt}$ atoms,

$$
\Delta(\mu / \rho)=(\mu / \rho)\left(E_{+}\right)-(\mu / \rho)\left(E_{-}\right),
$$

one obtains the total $\mathrm{Pt}$ loading of the sample,

$$
m_{\mathrm{Pt}}^{\mathrm{XAS}}=\frac{\Delta \mu D}{(\Delta \mu / \rho)}=w_{\mathrm{Pt}} \rho_{\mathrm{sa}} D_{\mathrm{sa}}=\rho_{\mathrm{Pt}}^{\mathrm{sa}} D_{\mathrm{sa}},
$$

where $\rho_{\mathrm{sa}}$ is the volume density of the sample, $E_{-}$is the energy immediately before the $\mathrm{Pt}$ absorption edge, $E_{+}$is the energy immediately after the $\mathrm{Pt}$ absorption edge, $w_{\mathrm{Pt}}$ is the weight percentage of $\mathrm{Pt}$ relative to the total sample weight, and $D_{\mathrm{sa}}$ is the sample thickness (Haubold et al., 1996).

1.1.8. Determination of the valence state. From the quantities $\mu D\left(E_{+}\right)$and $\mu D\left(E_{-}\right)$, one obtains the normalized absorption spectrum

$$
\mu D(E)_{\mathrm{norm}}=\frac{\mu D(E)-\mu D\left(E_{-}\right)}{\mu D\left(E_{+}\right)-\mu D\left(E_{-}\right)} .
$$
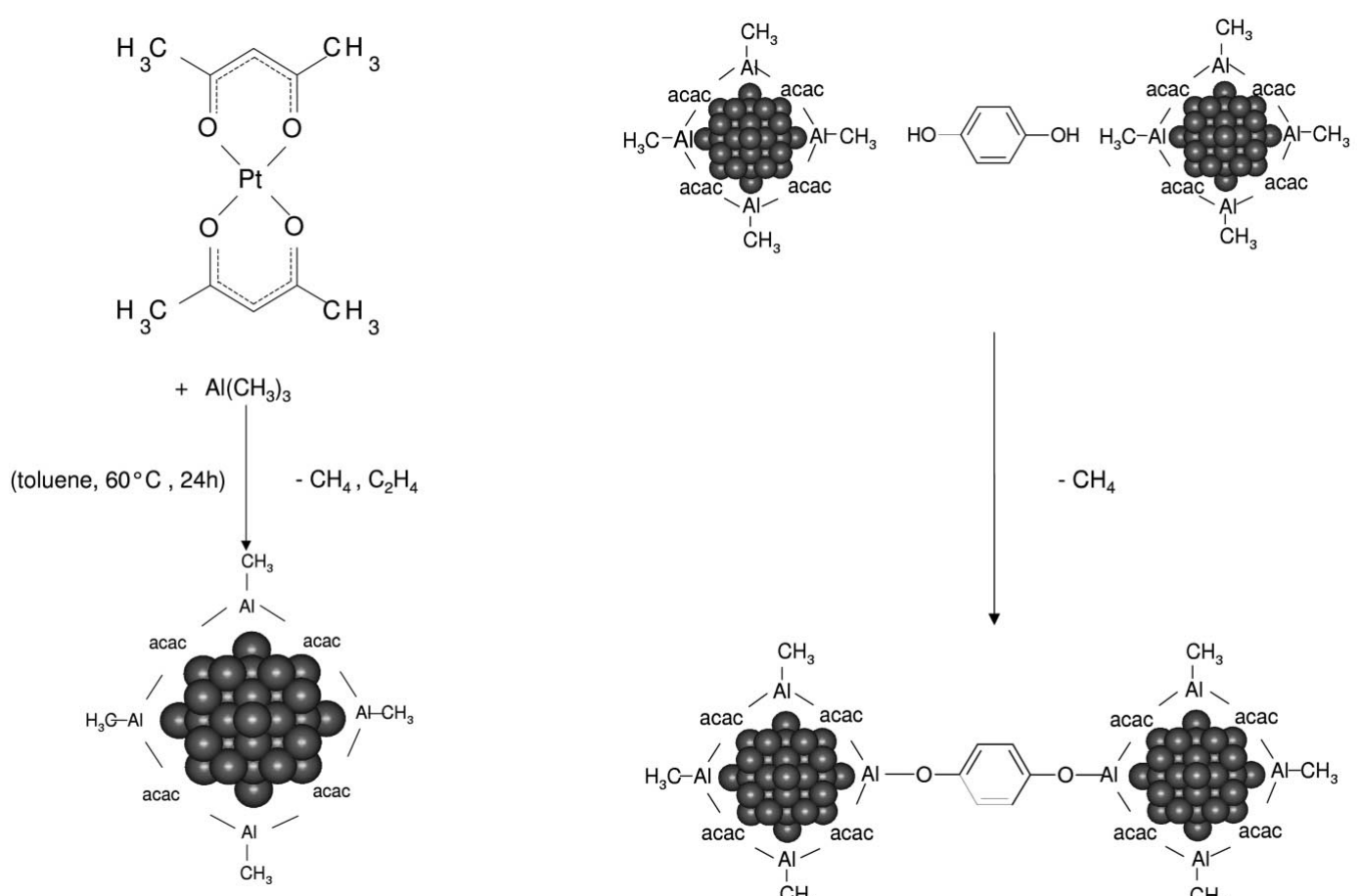

Figure 5

Principle of the synthesis of Al-organic-stabilized Pt colloids (left) and the protonolytic crosslinking mechanism (right). 


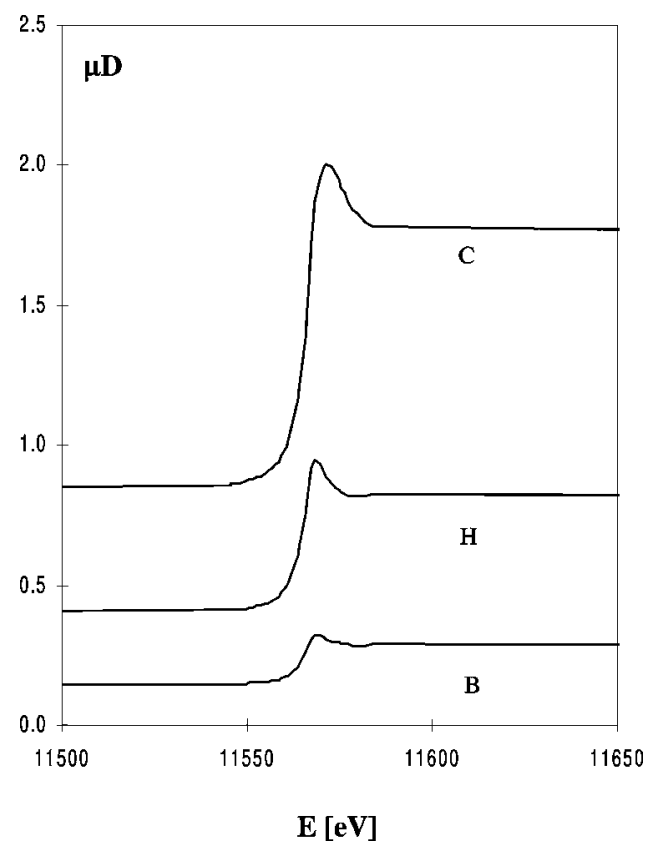

Figure 6

XAS spectra for Pt/hydroquinone (H), Pt/biphenyl (B) and the pure $\mathrm{Pt}$ colloid (C).

Thus, the absorption spectra of samples with different $\mathrm{Pt}$ content can be directly compared. Especially from the height of the white line (Brown et al., 1977; Mattheiss \& Dietz, 1980; Röhler, 1995), one obtains information on the valence state of the $\mathrm{Pt}$ atoms in the sample by comparison with a reference sample, e.g. a Pt foil.

\section{Experimental}

\subsection{Sample preparation}

Three Pt colloid samples were prepared at the MPI für Kohlenforschung: the first sample is a pure Pt colloid without any spacer molecules, the second one is a hybrid material with the Pt particles being interconnected by hydroquinone, and the third one a hybrid with 4,4'-dihydroxybiphenyl as spacer molecule. For the synthesis of these samples, the method of reductive stabilization of nanoparticles with aluminium trialkyls was used, a preparation method recently developed by Bönnemann and coworkers (Bönnemann et al., 1999).

This synthesis has proven to be a reliable method for the fabrication of small zero-valent transition-metal particles over a wide range of noble metals and magnetic elements, e.g. Pt or Co. The key feature of this synthesis is the formation of a reactive aluminium-organic protecting shell around the particles (see Fig. 5). Reactive Al-alkyl groups present in the shell open up the possibility of chemical reactions at the surface of a colloidal particle. One example is the substitution of organic groups at the $\mathrm{Al}$ atom in order to modify the dispersive properties of the colloid. If the substituents are bifunctional, e.g. diols like hydroquinone or 4,4'-dihydroxybiphenyl, a crosslinking of the particles can be brought about, which leads to the formation of a three-dimensional network (Fig. 5).

Thus, the interparticle distance can easily be controlled by variation of the type (i.e. the length) of the spacer molecule being used (Bönnemann et al., 2002).

2.1.1. Synthesis of colloidal Pt nanoparticles. Platinum acetylacetonate $(5 \mathrm{mmol})\left[\mathrm{Pt}(\mathrm{acac})_{2}\right]$ was dissolved under argon atmosphere in dry toluene $(200 \mathrm{ml})$. Trimethylaluminium $(20 \mathrm{mmol})\left[\mathrm{Al}\left(\mathrm{CH}_{3}\right)_{3}\right]$ was dissolved in toluene $(200 \mathrm{ml})$ and carefully added over $4 \mathrm{~h}$ at $333 \mathrm{~K}$. After $24 \mathrm{~h}$, when the gas evolution had stopped, the solution was filtered and all volatile components were completely evaporated in vacuum. In the residue, colloidal platinum powder $(2.4 \mathrm{~g})$ was obtained.

2.1.2. Formation of crosslinked Pt networks. The Pt colloid $(0.5 \mathrm{~g})$ was dissolved in dry tetrahydrofuran $(500 \mathrm{ml})$ (thf). The respective spacer molecule $(5 \mathrm{mmol})$ was dissolved in thf $(200 \mathrm{ml})$ and added dropwise to the colloidal solution. The mixture was stirred overnight at ambient temperature. The network precipitated and was filtered and washed with thf to remove excess spacer molecules.

2.1.3. ASAXS and XAS experiments. ASAXS and XAS measurements at X-ray energies near the $L_{3}$-absorption edge of $\mathrm{Pt}(E=11.564 \mathrm{keV})$ were performed on the three samples. Two X-ray detectors were used: a pin diode for the measurement of the incoming and transmitted primary intensities, $I_{0}$, and $I_{T}$ [for the determination of the absorption coefficient, $\left.\mu D \propto \ln \left(I_{T} / I_{0}\right)\right]$, and a two-dimensional positionsensitive multiwire gas proportional counter for the SAXS intensities. The XAS spectra were recorded in an energy interval of $11.0 \mathrm{keV} \leq E \leq 12.8 \mathrm{keV}$. The contrast-variation experiments for the $\mathrm{Pt} / \mathrm{hydroquinone}$ and the $\mathrm{Pt} / \mathrm{biphenyl}$ sample were performed at X-ray energies of $E_{1}=11.46 \mathrm{keV}$ and $E_{2}=11.55 \mathrm{keV}$. The SAXS data for the free Pt colloid were collected at energies $E_{1}=10.98 \mathrm{keV}$ and $E_{2}=11.55 \mathrm{keV}$. The maximum observable $Q$ range for all three samples and energies was $0.01 \AA^{-1} \leq Q \leq 0.63 \AA^{-1}$. Both the ASAXS and the XAS measurements were carried out at the JUSIFA beamline at DESY-HASYLAB (Haubold et al., 1989).

\section{Results and discussion}

\subsection{X-ray absorption spectroscopy}

The unnormalized XAS spectra for the three Pt colloid samples are shown in Fig. 6. As expected, the edge jumps $\Delta \mu D$, which are proportional to the Pt content in the sample, differ considerably from one another. With increasing spacermolecule length, we observe a decrease in $\Delta \mu D$. This finding already indicates that the interconnection of the Pt particles with the spacer molecules must have been successful to some extent, since the amount of Pt originally used for the synthesis was the same for all samples.

The normalized absorption spectra (Fig. 7) of the three samples, compared with that of a Pt foil, clearly show that the $\mathrm{Pt}$ nanoparticles are in a zero-valent state, since the heights of the white lines (HWL) are almost equal to one another [HWL 


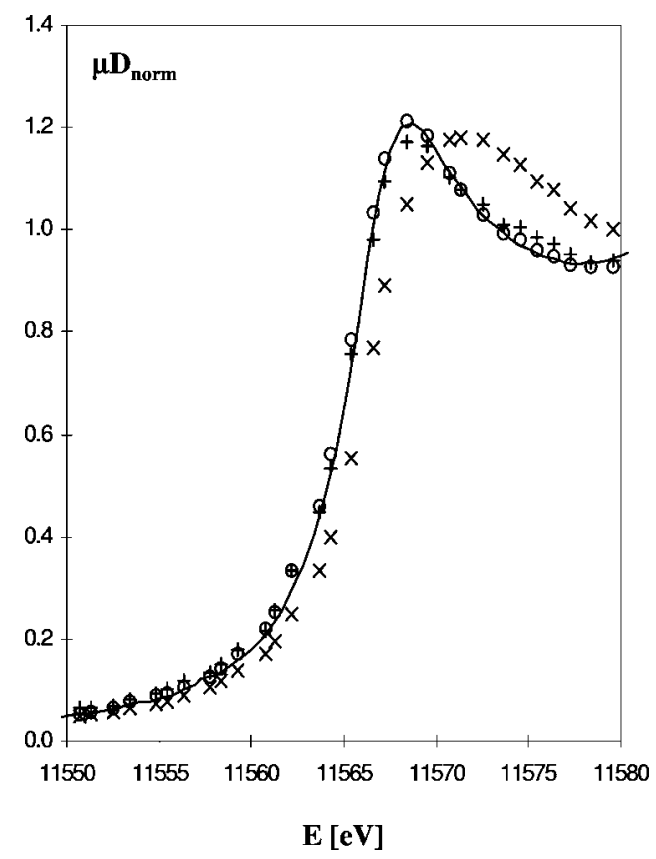

Figure 7

Normalized XAS spectra for Pt/hydroquinone ( $\circ)$, Pt/biphenyl (+), Pt colloid $(\times)$ and a Pt foil (solid line).

$\simeq 1.20$ (3) for all samples]. For the pure Pt colloid, we observe a small shift of the absorption edge $(\Delta E \simeq 1.0 \mathrm{eV})$ and a broadening of the white line. Both effects may be attributed to the presence of Pt-organic complexes, which are assumed to be produced at early stages of the reduction process, i.e. some of the Pt atoms are likely not to be contained in the nanoparticles, but in the surrounding matrix.

\subsection{Small-angle $X$-ray scattering}

The SAXS curves $\mathrm{d} \Sigma / \mathrm{d} \Omega\left(E_{1}\right)$ and $\mathrm{d} \Sigma / \mathrm{d} \Omega\left(E_{2}\right)$, and the corresponding differences $\mathrm{d} \Sigma^{*} / \mathrm{d} \Omega=\mathrm{d} \Sigma / \mathrm{d} \Omega\left(E_{1}\right)-$ $\mathrm{d} \Sigma / \mathrm{d} \Omega\left(E_{2}\right)$ for the three samples are depicted in Fig. 8. The most striking feature for all samples is, besides the interparticle correlation peak, the non-vanishing $Q^{-4}$ background after the subtraction, indicating that the samples are inhomogeneous. These inhomogeneities are caused by the particles themselves, which is easily shown by TEM investigations (see Fig. 9). Inside each sample, regions of particle agglomerates are found, which contain nearly all the (interconnected) Pt particles. The particle agglomerates are assumed to be surrounded by a matrix which consists of organic material (unused spacer molecules, excess of Al-organic shell material) and/or Pt-organic complexes (see previous section). This holds at least for the pure Pt colloid.

For the $\mathrm{Pt} /$ hydroquinone and $\mathrm{Pt} /$ biphenyl sample, the unused organic material should be removed by filtering and washing with thf (see \$2.1.2). In these cases, the samples can be considered as mesoporous systems. This assumption is in agreement with results of sorption measurements (Waldöfner, 2002), showing that the regions between the particle agglomerates are accessible. Since these large-scale inhomogeneities are beyond the scope of this study, the $Q^{-4}$ scattering contributions (Fig. 8) were subtracted from the difference scattering curves. For all further considerations, the backgroundcorrected particle scattering intensities,

$$
\frac{\mathrm{d} \Sigma}{\mathrm{d} \Omega_{p}}(Q)=\frac{\mathrm{d} \Sigma^{*}}{\mathrm{~d} \Omega}(Q)-a Q^{-4}
$$

(where $a$ is the Porod constant), are used.

3.2.1. Characteristic function. The most general information that can be directly obtained from the small-angle intensity curve is the so-called characteristic function, which is similar to the Patterson function in X-ray crystallography (Porod, 1951, 1982; Feigin \& Svergun, 1987),
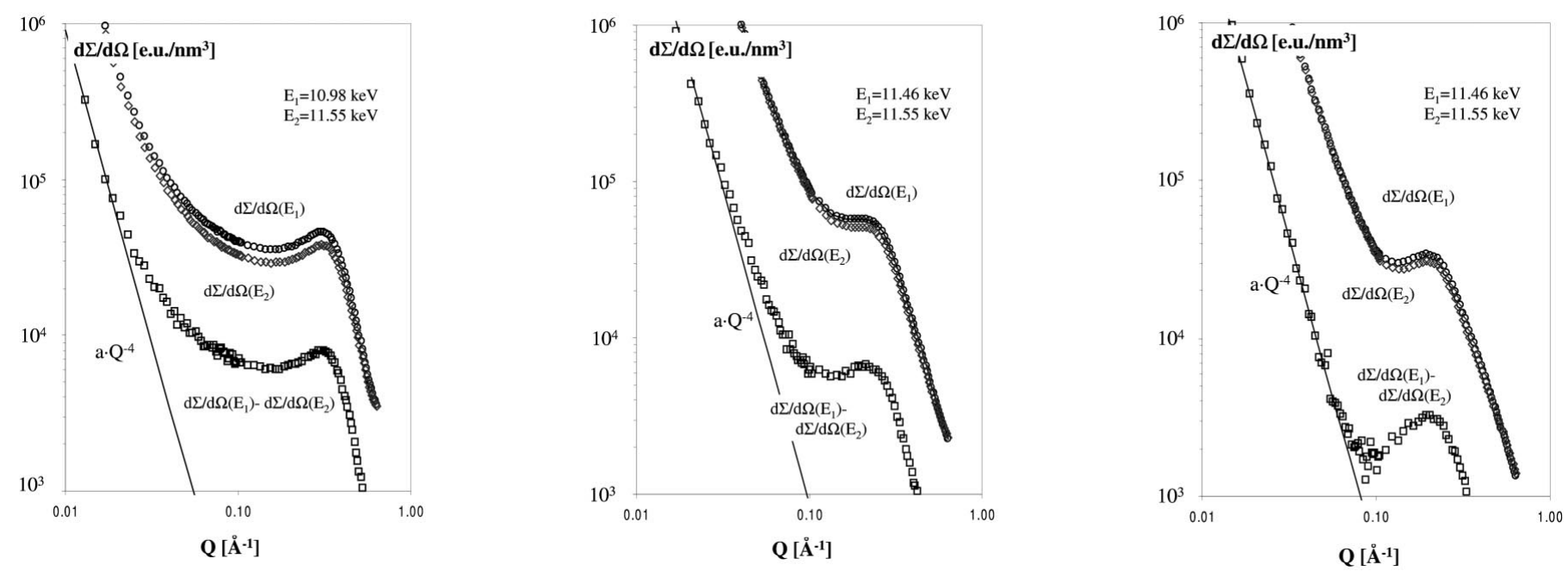

Figure 8

SAXS intensities at energies $E_{1}, E_{2}$, corresponding differences, and fitted $Q^{-4}$ scattering contributions (solid line) for Pt colloid (left), Pt/hydroquinone (middle) and Pt/biphenyl (right). 


\section{Table 1}

Radial parameters $R_{1}, R_{2}$, interparticle distance parameters $d, D_{0}$, and spacer lengths $l_{\mathrm{sp}}$ obtained from the radial Patterson functions and $l_{\mathrm{th}}$ from molecular modelling.

\begin{tabular}{lllllll}
\hline & $R_{1}(\AA)$ & $R_{2}(\AA)$ & $d(\AA)$ & $D_{0}(\AA)$ & $l_{\text {sp }}(\AA)$ & $l_{\text {th }}(\AA)$ \\
\hline Pt colloid & $6(1)$ & $23(1)$ & $17(1)$ & $5(2)$ & - & - \\
Pt/hydroquinone & $7(1)$ & $32(1)$ & $25(1)$ & $11(2)$ & $6(3)$ & 5.8 \\
Pt/biphenyl & $7(1)$ & $35(1)$ & $28(1)$ & $14(2)$ & $9(3)$ & 9.9 \\
\hline
\end{tabular}

$$
\gamma(r)=\frac{1}{Q_{0}} \int_{0}^{\infty} \frac{\mathrm{d} \Sigma}{\mathrm{d} \Omega_{p}}(Q) \frac{\sin (Q r)}{Q r} Q^{2} \mathrm{~d} Q,
$$

with the invariant

$$
Q_{0}=\int_{0}^{\infty} \frac{\mathrm{d} \Sigma}{\mathrm{d} \Omega_{p}}(Q) Q^{2} \mathrm{~d} Q .
$$

In this study, the radial Patterson function (Porod, 1948)

$$
\alpha(r)=r^{2} \gamma(r)
$$

was used to obtain information on particle sizes and interparticle distances. In order to avoid series termination effects, the wide-angle part of each scattering curve $\left(0.55 \AA^{-1} \leq Q \leq\right.$ $0.63 \AA^{-1}$ ) was fitted by the two-parameter model function

$$
\frac{\mathrm{d} \Sigma}{\mathrm{d} \Omega_{p}}(Q) Q^{4}=a+b Q^{4} .
$$

The constant background $b$ was subtracted from the particle scattering curves and the scattering intensities beyond $Q_{\max }=$ $0.63 \AA^{-1}$ were calculated by the formula

$$
\frac{\mathrm{d} \Sigma}{\mathrm{d} \Omega_{p}}(Q)=a Q^{-4}
$$

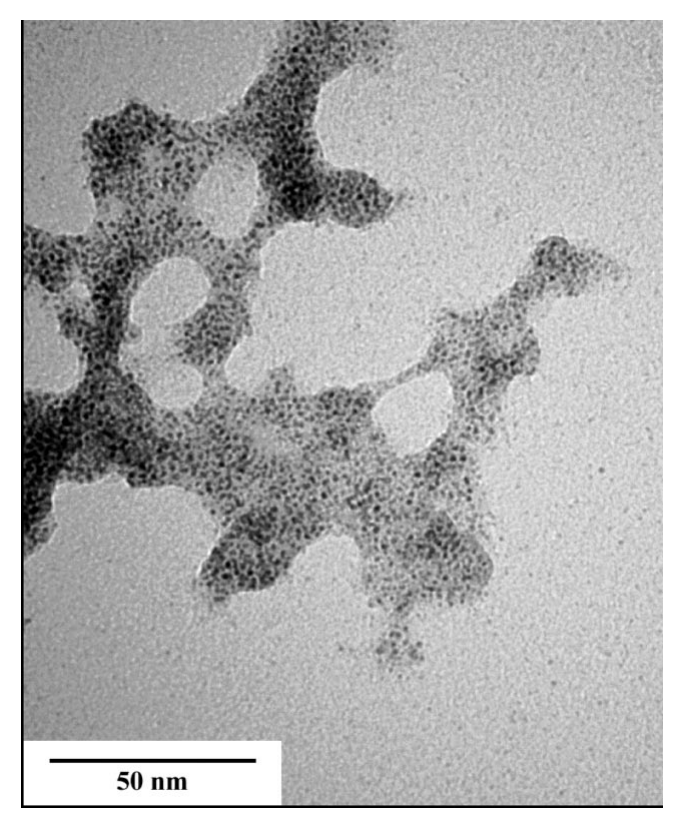

Figure 9

TEM image of Pt nanoparticle agglomerates. These structures can reach sizes of up to $1 \mu \mathrm{m}$.
The numerical integration for each $r$ value was performed up to $Q=1000 \AA^{-1}$.

The radial Patterson functions for the three samples are shown in Fig. 10 and the values for the particle radii $R_{1}$, interparticle distance parameters $d, D_{0}$, and spacer lengths $l_{\mathrm{sp}}$ [see equation (29)], along with the spacer lengths $l_{\text {th }}$ obtained from molecular-modelling calculations using the program SYBYL (2000), are given in Table 1.

The particle radii are simply given by the corresponding maxima of the first peaks of the $\alpha(r)$ at $r=R_{1}$. Together with the maxima of the second peaks (at $r=R_{2}$ ), the interparticle distances $d=R_{2}-R_{1}$, and the parameters $D_{0}=d-2 R_{1}$ are readily obtained. The error estimates for $R_{1}$ and $R_{2}$ were chosen such that the differences in the particle radii are insignificant with respect to the standard deviations $\sigma$, since the Pt nanoparticles used for all samples originate from the same synthesis. A value fulfilling this criterion is $\sigma=1 \AA$. The $\sigma$ estimates for the other parameters were calculated by error propagation. The results for the particle radii agree well with the one obtained from the TEM analysis $(R=5.3 \AA$; see Fig. $3)$. The interparticle distances $d$ and the parameters $D_{0}$ are found to be consistent with the different types of spacer molecules used for the interconnection of the metal particles.

For the pure Pt colloid, the Pt particles are not interconnected by spacer molecules; however, the analysis yields a $D_{0}$ value of $5 \AA$. This value is twice the thickness of the Alorganic protecting shell which surrounds each of the $\mathrm{Pt}$ particles $\left(D_{0}=2 t_{\mathrm{ps}}\right)$. This result can easily be crosschecked by subtracting $2 t_{\mathrm{ps}}$ from the $D_{0}$ values of the other two samples. The differences, $l_{\mathrm{sp}}=D_{0}-2 t_{\mathrm{ps}}=6 \AA$ for Pt/hydroquinone and $l_{\mathrm{sp}}=9 \AA$ for Pt/biphenyl, are consistent with the theoretical spacer lengths of $l_{\text {th }}=5.8 \AA$ and $l_{\text {th }}=9.9 \AA$ calculated by molecular modelling. This finding also indicates that the metal particles are completely interconnected by the spacers.

3.2.2. Least-squares model. The least-squares refinements were performed using the model function according to equa-

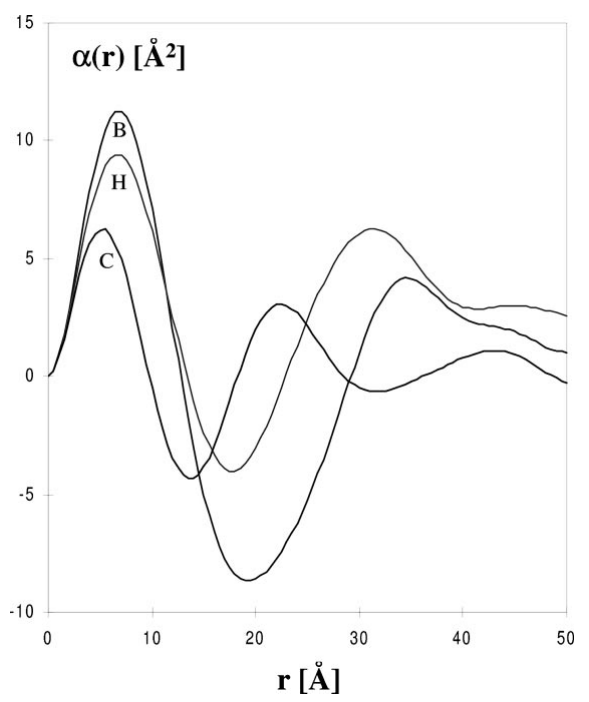

Figure 10

Radial Patterson functions for Pt/biphenyl (B), Pt/hydroquinone (H) and Pt colloid (C). 
Table 2

Residual index $R_{w}$, refined model parameters $R_{0}, \sigma_{0}, D_{0}$, and calculated spacer lengths $l_{\text {sp }}$.

\begin{tabular}{llllrl}
\hline & $R_{w}$ & $R_{0}(\AA)$ & $\sigma_{0}$ & $D_{0}(\AA)$ & $l_{\text {sp }}(\AA)$ \\
\hline Pt colloid & 0.034 & $5.5(3)$ & $0.27(1)$ & $3.7(8)$ & - \\
Pt/hydroquinone & 0.028 & $5.3(3)$ & $0.26(2)$ & $9.8(8)$ & $6(1)$ \\
Pt/biphenyl & 0.032 & $6.2(2)$ & $0.17(3)$ & $12.3(4)$ & $8.6(9)$ \\
\hline
\end{tabular}

tions (17) and (36). The scattering contrast $\Delta(n f)$ was chosen as an additional parameter for two reasons.

The non-vanishing $Q^{-4}$ scattering contributions indicate that all samples are inhomogeneous; however, the model function is only valid for a homogeneous sample (fluid model). In the case of an inhomogeneous sample, the concentration parameter $c_{0}$ will describe the local concentration of the $\mathrm{Pt}$ particles inside the region of particle agglomerates (if the scattering contrast is a free parameter; otherwise the refinement is likely to fail). The particle scattering intensity $\left[\propto c_{\mathrm{gl}} \Delta(n f)^{2}\right]$, however, is a global quantity (valid for the whole sample), which would require a second concentration parameter $c_{\mathrm{gl}} \leq c_{0}$ to be introduced into the model. Since, in addition, the particle and matrix densities were not known (and in order to avoid parameter correlation effects), the model was not changed. Therefore, the refined square of the scattering contrast will yield the quantity

$$
\Delta(n f)_{\mathrm{LSQ}}^{2}=\frac{c_{\mathrm{gl}}}{c_{0}} \Delta(n f)_{\text {true }}^{2}
$$

For the least-squares refinements, the weighted crystallographic residual index,

$$
R_{w}=\left\{\frac{\sum_{k=1}^{n_{\mathrm{obs}}}\left[\mathrm{d} \Sigma / \mathrm{d} \Omega_{p}\left(Q_{k}\right)-\mathrm{d} \Sigma / \mathrm{d} \Omega\left(Q_{k}\right)_{\mathrm{LSQ}}\right]^{2} / \sigma_{k}^{2}}{\sum_{k=1}^{n_{\mathrm{obs}}} \mathrm{d} \Sigma / \mathrm{d} \Omega_{p}\left(Q_{k}\right)^{2} / \sigma_{k}^{2}}\right\}^{1 / 2}
$$

where the $\sigma_{k}$ are the standard deviations of the observed intensities $\mathrm{d} \Sigma / \mathrm{d} \Omega_{p}\left(Q_{k}\right)$, was used as an indicator for the agreement between observed and model intensities (see Table 2).

The separated particle scattering curves along with their refined model functions and the resulting log-normal particle size distributions $P(R)$ are shown in Fig. 11, and the average structure factors $\beta(Q)=[\Delta(Q)]^{-1}$ [see equation (19)] are depicted in Fig. 12.

From the average structure factors, the radial distribution functions $g(r)$ [see equation (8)] were determined by Fourier transformation (Fig. 12):

$$
g(r)=1+\frac{1}{2 \pi^{2} c_{0}} \int_{0}^{\infty}[\beta(Q)-1] \frac{\sin (Q r)}{Q r} Q^{2} \mathrm{~d} Q .
$$

Finally, formal coordination numbers $Z$, i.e. estimates for the average number of successful interconnections per particle, were obtained by integration of the $g(r)$ over the volume of the first coordination shell:

$$
Z=4 \pi c_{0} \int_{r_{0}-\Delta}^{r_{0}+\Delta} g(r) r^{2} \mathrm{~d} r
$$

with $g\left(r_{0}\right)=\max ; 2 \Delta$ is the total width of the coordination peak.

Since the coordination peaks for all samples are not as clearly defined as in the case for uniformly sized particles, e.g. liquid metals (Waseda, 1980), the approximation

$$
Z \simeq 8 \pi c_{0} \int_{r_{0}-\Delta}^{r_{0}} g(r) r^{2} \mathrm{~d} r
$$

was chosen for the determination of the coordination numbers.

The least-squares refinement results for all samples, together with additional quantities derived from the model para-

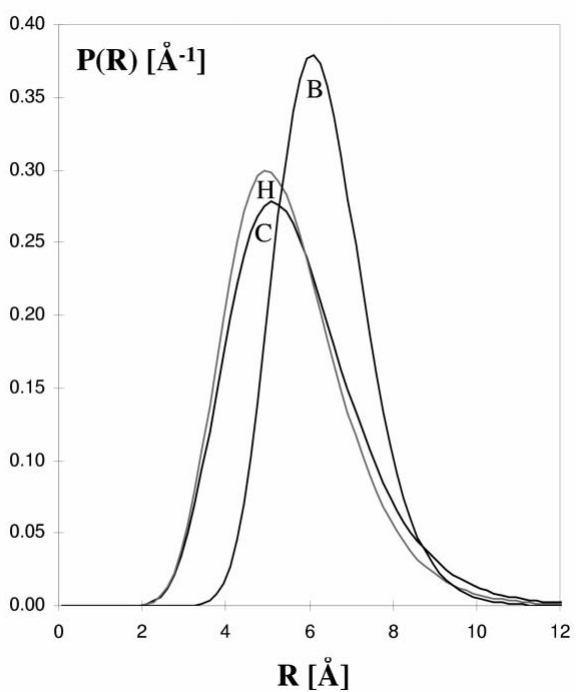

\section{Figure 11}

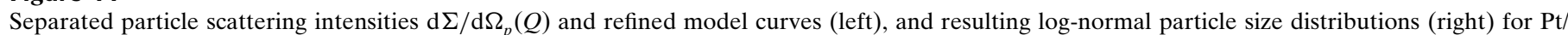
biphenyl (B), Pt colloid (C) and Pt/hydroquinone (H). 
Table 3

Refined model parameters $c_{0}$ and $\Delta(n f)_{\text {LSQ }}^{2}$, calculated concentration parameters $c_{\mathrm{gl}}$, theoretical scattering contrasts $\Delta(n f)_{\text {th }}^{2}$ for metallic platinum, and products $c_{0} \Delta(n f)_{\mathrm{LSQ}}^{2}, c_{\mathrm{gl}} \Delta(n f)_{\mathrm{th}}^{2}$.

\begin{tabular}{lllllll}
\hline & $c_{0}\left(10^{-3} \AA^{-3}\right)$ & $\Delta(n f)_{\mathrm{LSQ}}^{2}\left(10^{3}\right.$ e.u. $\left.\AA-6\right)$ & $c_{\mathrm{gl}}\left(10^{-5} \AA^{-3}\right)$ & $\Delta(n f)_{\mathrm{th}}^{2}\left(10^{3}\right.$ e.u. $\left.\AA^{-6}\right)$ & $c_{0} \Delta(n f)_{\mathrm{LSQ}}^{2}\left(10^{3}\right.$ e.u. $\left.\AA^{-9}\right)$ & $c_{\mathrm{gl}} \Delta(n f)_{\mathrm{th}}^{2}\left(10^{3}\right.$ e.u. $\left.\AA^{-9}\right)$ \\
\hline Pt colloid & $0.164(6)$ & $116(17)$ & $0.82(2)$ & 4160 & $0.0191(29)$ & $0.0340(8)$ \\
Pt/hydroquinone & $0.042(2)$ & $233(43)$ & $0.43(1)$ & 2180 & $0.0097(18)$ & $0.0093(3)$ \\
Pt/biphenyl & $0.022(2)$ & $125(16)$ & $0.11(1)$ & 2180 & $0.0028(4)$ & $0.0023(1)$ \\
\hline
\end{tabular}

\section{Table 4}

Forward scattering $\mathrm{d} \Sigma / \mathrm{d} \Omega_{p}(0)$ in the case of negligible interparticle correlation, average Pt particle volume $\left\langle V_{p}\right\rangle$, average of squared particle volume $\left\langle V_{p}^{2}\right\rangle$, Pt loadings $m_{\mathrm{Pt}}^{\mathrm{XAS}}$ obtained from XAS, $m_{\mathrm{Pt}}^{\mathrm{LSQ}}$ calculated from $\mathrm{d} \Sigma / \mathrm{d} \Omega_{p}(0)$, and coordination numbers $Z$.

\begin{tabular}{|c|c|c|c|c|c|c|}
\hline & $\mathrm{d} \Sigma / \mathrm{d} \Omega_{p}(0)\left(10^{4}\right.$ e.u. $\left.\mathrm{nm}^{-3}\right)$ & $\left\langle V_{p}\right\rangle\left(10^{3} \AA^{3}\right)$ & $\left\langle V_{p}^{2}\right\rangle\left(10^{6} \AA^{6}\right)$ & $m_{\mathrm{Pt}}^{\mathrm{XAS}}\left(10^{-3} \mathrm{~g} \mathrm{~cm}^{-2}\right)$ & $m_{\mathrm{Pt}}^{\mathrm{LSQ}}\left(10^{-3} \mathrm{~g} \mathrm{~cm}^{-2}\right)$ & $Z$ \\
\hline Pt colloid & $3.48(52)$ & 0.975 & 1.842 & $8.44(25)$ & $4.69(70)$ & $5.97(30)$ \\
\hline $\mathrm{Pt} /$ hydroquinone & $1.29(24)$ & 0.852 & 1.331 & $3.86(12)$ & $4.03(74)$ & $3.58(18)$ \\
\hline Pt/biphenyl & $0.49(12)$ & 1.166 & 1.770 & $1.31(4)$ & $1.57(20)$ & $2.10(11)$ \\
\hline
\end{tabular}
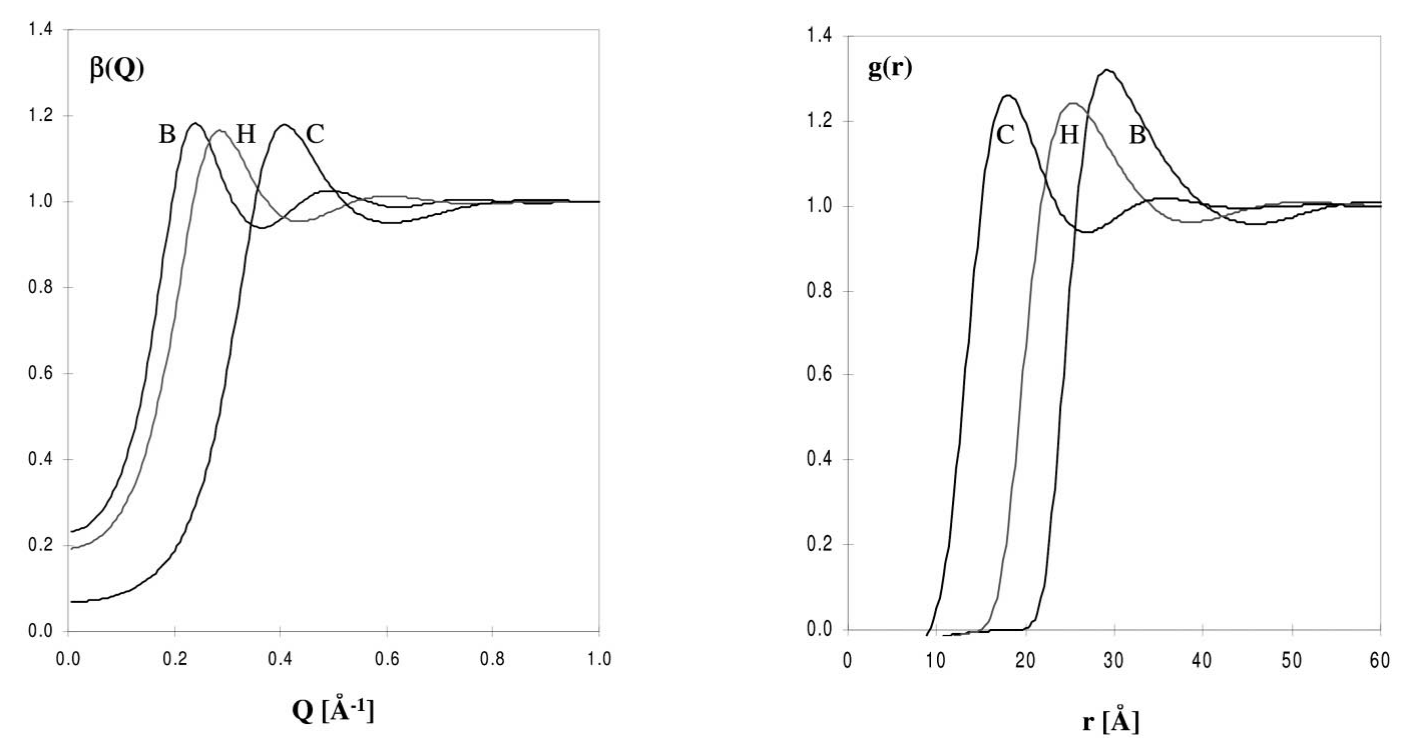

Figure 12

Average structure factors $\beta(Q)$ (left) and radial distribution functions $g(r)$ (right) for Pt/biphenyl (B), Pt colloid (C) and Pt/hydroquinone (H).

meters and X-ray absorption measurements, are given in Tables 2-4.

(a) Particle radii and interparticle distance parameters. As expected, the results for the most frequently occurring radius $R_{0}$ for the three investigated samples are found to be consistent with the values determined from the radial Patterson functions $\alpha(r)$, and, moreover, they are in excellent agreement with the result obtained from the TEM analysis $\left(R_{0}=5.3 \AA\right)$, except for the $\mathrm{Pt} /$ biphenyl sample, where the refinement yields a somewhat larger value of $R_{0} \simeq 6 \AA$. The distance parameters $D_{0}$ and the resulting spacer lengths $l_{\text {sp }}$ are consistent with the spacer lengths obtained from the radial Patterson functions as well as with the spacer lengths from molecular-modelling calculations (Table 1).

The refined $D_{0}$ parameter for the pure $\mathrm{Pt}$ colloid $\left(D_{0}=2 t_{\mathrm{ps}}\right)$ yields a value of approximately $4 \AA$, i.e. the thickness of the Al-organic protecting shell is about $2 \AA$, which corresponds roughly to the diameter of only one $\mathrm{CH}_{3}$ group. This finding implies that the major part of the Al- organic material must form some kind of alloy on the particle surface, together with the Pt atoms.

(b) Scattering contrasts, concentration parameters and Pt loadings. From the Pt loadings

$$
m_{\mathrm{Pt}}^{\mathrm{XAS}}=\Delta \mu D / \Delta(\mu / \rho)_{\mathrm{Pt}}
$$

[with $\Delta(\mu / \rho)_{\mathrm{Pt}}=118.04 \mathrm{~cm}^{2} \mathrm{~g}^{-1}$ ], and the average particle volume

$$
\left\langle V_{p}\right\rangle=\frac{4 \pi}{3} \int_{0}^{\infty} P(R) R^{3} \mathrm{~d} R,
$$

approximate global concentration parameters $c_{\mathrm{gl}}$ can be determined. Using the assumptions that all $\mathrm{Pt}$ is contained in the particles and the density of the $\mathrm{Pt}$ particles is $\rho_{\mathrm{Pt}}=$ $21.2 \mathrm{~g} \mathrm{~cm}^{-3}$, i.e. the density of metallic $\mathrm{Pt}$, the $c_{\mathrm{gl}}$ are given by

$$
c_{\mathrm{gl}}=m_{\mathrm{Pt}}^{\mathrm{XAS}} /\left\langle V_{p}\right\rangle \rho_{\mathrm{Pt}} D_{\mathrm{sa}}
$$


where $D_{\mathrm{sa}}=500 \mu \mathrm{m}$ is the sample thickness (for all samples). As can be seen in Table 3, the $c_{\mathrm{gl}}$ are much smaller than the refined (local) concentration parameters $c_{0}$. This finding shows that the spatial distributions of the particles in the samples are indeed strongly inhomogeneous, and serves as an (additional) explanation for the remaining $Q^{-4}$ scattering contributions in the $\mathrm{d} \Sigma / \mathrm{d} \Omega^{*}(Q)$.

On the other hand, approximate values $m_{\mathrm{Pt}}^{\mathrm{LSQ}}$, i.e. the amount of Pt contained in the particles, can be calculated from the forward scattering

$$
\begin{aligned}
\frac{\mathrm{d} \Sigma}{\mathrm{d} \Omega_{p}}(0) & =c_{0} \Delta(n f)_{\mathrm{LSQ}}^{2}\left\langle V_{p}^{2}\right\rangle \\
& \simeq c_{\mathrm{g} 1} \Delta(n f)_{\mathrm{th}}^{2}\left\langle V_{p}^{2}\right\rangle \\
& =\frac{m_{\mathrm{Pt}}^{\mathrm{LSQ}}}{\left\langle V_{p}\right\rangle \rho_{\mathrm{Pt}} D_{\mathrm{sa}}} \Delta(n f)_{\mathrm{th}}^{2}\left\langle V_{p}^{2}\right\rangle,
\end{aligned}
$$

where

$$
\left\langle V_{p}^{2}\right\rangle=\frac{16 \pi^{2}}{9} \int_{0}^{\infty} P(R) R^{6} \mathrm{~d} R
$$

is the average square of the particle volume. Here it is assumed that the particles are homogeneously distributed over the whole sample, i.e. the interparticle correlation is negligible, and the scattering contrast is given by

$$
\Delta(n f)_{\mathrm{th}}^{2}=\frac{1}{\Omega_{\mathrm{Pt}}^{2}} \Delta f_{\mathrm{Pt}}^{2},
$$

where $\Omega_{\mathrm{Pt}}=15.1 \AA^{3}$ is the volume of a Pt atom, and $\Delta f_{\mathrm{th}}^{2}$ is the difference of the squared atomic form factors of $\mathrm{Pt}$ at two different energies $E_{1}$ and $E_{2}$ :

$$
\begin{aligned}
\Delta f_{\mathrm{th}}^{2}= & {\left[f_{0}+f^{\prime}\left(E_{1}\right)\right]^{2}-\left[f_{0}+f^{\prime}\left(E_{2}\right)\right]^{2} } \\
& +f^{\prime \prime}\left(E_{1}\right)^{2}-f^{\prime \prime}\left(E_{2}\right)^{2},
\end{aligned}
$$

where $f_{0}=78$ electrons, and $f^{\prime}(E), f^{\prime \prime}(E)$ are anomalous dispersion corrections from Cromer \& Liberman (1981) calculations, i.e. the scattering contribution from the matrix is in this case also considered to be negligible.

The Pt loadings $m_{\mathrm{Pt}}^{\mathrm{LSQ}}$ for the Pt/hydroquinone and the Pt/ biphenyl sample are close to the values obtained from the X-ray absorption measurements (see Table 4), thus showing that for these two samples all $\mathrm{Pt}$ is contained in the particles. The products $c_{0} \Delta(n f)_{\mathrm{LSQ}}^{2}$ and $c_{\mathrm{gl}} \Delta(n f)_{\mathrm{th}}^{2}$ serve as crosschecks for the validity of the assumptions made for the calculation of the $m_{\mathrm{Pt}}^{\mathrm{LSQ}}$ and the $c_{\mathrm{gl}}$, and agree very well with each other. This, however, is not the case for the pure Pt colloid. The products $c_{0} \Delta(n f)_{\text {LSQ }}^{2}$ and $c_{\mathrm{gl}} \Delta(n f)_{\text {th }}^{2}$ exhibit considerable differences and indicate that the assumption of a negligible matrixscattering contribution is invalid. This is more clearly demonstrated by the discrepancies between the Pt loadings $m_{\mathrm{Pt}}^{\mathrm{LSQ}}$ and $m_{\mathrm{Pt}}^{\mathrm{XAS}}$, showing that only $55 \%$ of the Pt material is contained in the particles. The result is supported by the different behaviour of the XAS spectrum (white-line broadening, shifted absorption edge) compared with the spectra of $\mathrm{Pt} /$ hydroquinone, Pt/biphenyl and the Pt foil.
One more important conclusion that can be drawn from these considerations is that the $\mathrm{Pt}$ particles appear to be genuine metal particles. This finding is supported by the good agreement of the products $c_{0} \Delta(n f)_{\mathrm{LSQ}}^{2}$ and $c_{\mathrm{gl}} \Delta(n f)_{\mathrm{th}}^{2}$, and corroborated by the normalized XAS spectra for Pt/hydroquinone and Pt/biphenyl, which are similar to the spectrum of the Pt foil. Since, as already mentioned, the Pt particles of all samples stem from the same synthesis, this conclusion holds also for the pure Pt colloid.

(c) Coordination numbers. From the coordination numbers, one usually obtains more detailed information on the local structure of the nanoparticle networks, which may be used for the creation of more advanced structure models. In the present study, the coordination numbers obtained from equation (51) will probably not yield correct values, since the particle coordination will be heavily (and non-trivially) influenced by the size polydispersity. Although the coordination numbers will not be correct (on an absolute scale), a (relative) comparison between the three samples is still possible, since the particle sizes and size distributions do not differ too much from one another (see Table 2).

In general, a decrease of the particle coordination with increasing spacer length is observed (see Table 4). For the pure Pt colloid, we obtain the largest coordination number, which is close to $Z=6$. The value for the $\mathrm{Pt} /$ hydroquinone sample of $Z \simeq 3.6$ indicates a lower local symmetry compared with the pure Pt colloid. The lowest coordination number, $Z \simeq$ 2 , is found for the Pt/biphenyl sample.

The results, especially for the Pt/biphenyl sample, clearly show that the coordination numbers cannot be (absolutely) correct, since a value of $Z=2$ corresponds to chain-like structures. If they were really chain-like objects, the low- $Q$ tail of the scattering curve should have a power between -1 and -2 , as one finds for stiff rods or flexible polymers. This, however, is definitely not the case (see Fig. 8).

Nevertheless, the coordination numbers show that the number of successful interconnections per particle is obviously decreasing with increasing spacer length. This finding may be explained by a growing mutual interference of the spacer molecules, which complicates the interconnection process.

\section{Conclusion}

The results obtained so far demonstrate that the nanostructures of three-dimensional metal-particle networks formed by crosslinking metal nanoparticles with organic spacer molecules can be investigated in ASAXS experiments. It was shown that the ASAXS method allows one to distinguish between inhomogeneities in the matrix (e.g. pore scattering) and inhomogeneities caused by the particles, e.g. by formation of particle agglomerates. For the structural characterization of the nanoparticle systems, a model function based on Vrij's analytical solution for a polydisperse collection of hard spheres was proposed and shown to yield reliable results concerning the analysis of particle size distributions and interparticle correlation effects. In combination with XAS 
measurements, which have to be carried out prior to an ASAXS experiment, a full quantitative analysis is possible.

The method of reactive interconnection of Al-organicstabilized metal particles by bifunctional spacers is found to work properly. However, the non-zero parameters $\sigma_{0}$ for the widths of the particle size distributions (see Table 2) reveal the main problem that has to be solved in order to increase the degree of particle ordering. The synthesis method has to be improved to produce nearly monodisperse particles with a uniform size and shape. This is the basic requirement for the creation of highly ordered nanolattices.

Our thanks are owed to Dr B. Tesche, Department of Electron Microscopy, Max-Planck-Institut für Kohlenforschung, for the TEM analysis of the Pt colloid samples.

\section{References}

Baxter, R. J. (1970). J. Chem. Phys. 52, 4559-4562.

Blum, L. \& Stell, G. (1979). J. Chem. Phys. 71, 42-46.

Bönnemann, H., Brijoux, W., Brinkmann, R., Endruschat, U., Hofstadt, W. \& Angermund, K. (1999). Rev. Roum. Chim. 11-12, 1003-1010.

Bönnemann, H., Waldöfner, N., Haubold, H.-G. \& Vad, T. (2002). Chem. Mater. 14, 1115-1120.

Brown, M., Peierls, R. E. \& Stern, A. (1977). Phys. Rev. B, 15, 738744.

Brust, M., Bethell, D., Schiffrin, D. J. \& Kiely, C. J. (1995). Adv. Mater. 7, 795-797.

Connolly, S. \& Fitzmaurice, D. (1999). Adv. Mater. 11, 1202-1205.

Cromer, D. T. \& Liberman, D. A. (1981). Acta Cryst. A37, 267-268.

Feigin, L. A. \& Svergun, D. I. (1987). Structure Analysis by Small Angle X-ray and Neutron Scattering, pp. 38-40. New York: Plenum Press.

Fournet, G. (1955). As cited in Small Angle Scattering of X-rays, by A. Guinier \& G. Fournet, p. 65. New York: Wiley.

Haubold, H.-G., Gebhardt, R., Buth, G. \& Goerigk, G. (1994). Resonant Anomalous X-ray Scattering, Theory and Applications, edited by G. Materlik, C. J. Sparks \& K. Fischer, pp. 295-304. Oxford: Elsevier Science.

Haubold, H.-G., Grünhagen, K., Wagener, M., Jungbluth, H., Heer, H., Pfeil, A., Rongen, H., Brandenburg, G., Möller, R., Matzerath, J., Hiller, P. \& Halling, H. (1989). Rev. Sci. Instrum. 60, 1943-1946.

Haubold, H.-G. \& Wang, X. H. (1995). Nucl. Instrum. Methods Phys. Res. B, 97, 50-54.

Haubold, H.-G., Wang, X. H., Jungbluth, H., Goerigk, G. \& Schilling, W. (1996). J. Mol. Struct. 383, 283-289.

Korgel, B. A., Fullam, S., Connolly, S. \& Fitzmaurice, D. (1998). J. Phys. Chem. B, 102, 8379-8388.

Kruif, C. G. de, Briels, W., May, R. P. \& Vrij, A. (1988). Langmuir, 4, 668-678.

Materlik, G., Sparks, C. J. \& Fischer, K. (1994). Editors. Resonant Anomalous X-ray Scattering, Theory and Applications. Oxford: Elsevier Science.

Mattheiss, L. F. \& Dietz, R. E. (1980). Phys. Rev. B, 22, 1663-1676.

Motte, L., Billoudet, F., Lacaze, E., Doulin, J. \& Pileni, M. (1997). J. Phys. Chem. B, 101, 138-144.

Pedersen, J. S. (1993). Phys. Rev. B, 47, 657-665.

Pedersen, J. S. (1994). J. Appl. Cryst. 27, 595-608.

Pedersen, J. S., Horsewell, A. \& Eldrup, M. (1996). J. Phys. Condens. Matter, 8, 8431-8455.

Porod, G. (1948). Acta Phys. Aust. 3, 255-292.

Porod, G. (1951). Kolloid-Z. 124, 83-114.

Porod, G. (1982). Small Angle X-ray Scattering, edited by O. Glatter \& O. Kratky, pp. 20-46. New York: Academic Press.

Röhler, J. (1995). J. Magn. Magn. Mater. 47/48, 175-180.

Salacuse, J. J. \& Stell, G. (1982). J. Chem. Phys. 77, 3714-3725.

Sequeira, A. D., Calderon, H. A., Kostorz, G. \& Pedersen, J. S. (1995). Acta Metall. Mater. 43, 3427-3439.

SYBYL (2000). Version 6.7.1. Molecular Modelling Software, Tripos Inc., 1699 South Hanley Rd, St. Louis, Missouri, 63144, USA.

Vrij, A. (1978). J. Chem. Phys. 69, 1742-1747.

Vrij, A. (1979). J. Chem. Phys. 71, 3267-3270.

Waldöfner, N. (2002). Aluminiumorganisch stabilisierte Übergangsmetallkolloide - Synthese, Bildungsmechanismus und Aufbau von organisierten Strukturen, thesis, RWTH Aachen (in preparation).

Waseda, Y. (1980). The Structure of Non-Crystalline Materials. New York: McGraw Hill. 